\title{
Synthesis and deformation mechanics of gradient nanostructured materials
}

\author{
Qingsong Pan \& Lei Lu ${ }^{*}$ \\ Shenyang National Laboratory for Materials Science, Institute of Metal Research, Chinese Academy of Sciences, Shenyang 110016, China \\ *Corresponding author (email: 1lu@imr.ac.cn)
}

Received 30 December 2021; Accepted 14 January 2022; Published online 24 April 2022

\begin{abstract}
The emerging gradient nanostructured metals and alloys containing spatially graded structural components with large variations in length scale and/or mechanical properties exhibit unprecedented mechanical performance. This perspective delineates the basic structural features of gradient nanostructures, i.e., structural components and spatial gradients, as well as related synthesis methods, excellent tensile properties, and novel deformation mechanisms. The challenges and prospect for the development of gradient nanostructured materials in the future are also addressed.
\end{abstract}

Keywords: gradient nanostructure, spatial gradient, synthesis, mechanical property, work hardening, deformation mechanism

Achieving both strength and ductility at the same time is a long-lasting pursuit for metallic materials. Inspired by biological materials in nature with spatial gradients in local chemical composition or constituents and/or structural characteristics at the nanoscale, which generally exhibit a good combination of strength and toughening, spatial gradients have been introduced into metallic materials [1]. The broad tunability of the gradient nanostructure (GNS) opens new frontiers to the development of high-performance metals and alloys for more challenging applications. In recent years, the emerging GNS metals and alloys have received growing attention of the materials community due to their unprecedented mechanical properties, such as superior strength and ductility combination, enhanced fatigue resistance compared with non-gradient counterparts [2-8].

Large amounts of experimental, computational, and theoretical studies have been devoted to investigating the design, fabrication, and mechanical performance of several gradient nanostructured metals and alloys [14,9-11]. Despite the rapid advance made in the past decade, a fundamental understanding of the mechanical behavior of GNS metallic materials is still in its infancy. In this perspective, we reviewed the basic features of gradient nanostructures, tensile properties, and novel deformation mechanisms. In addition, the insights on the challenges and opportunities in the field of GNS metals and alloys are provided.

As a typical hierarchical nanostructure, the two salient features of the gradient nanostructure are (1) structural components, and (2) continuous/discontinuous but ordered spatial distributions. Basically, the structural components may include the grain size, twin or lamellar thickness, dislocation density or pattern

(C) The Author(s) 2022. Published by China Science Publishing \& Media Ltd. and EDP Sciences. This is an Open Access article distributed under the terms of the Creative Commons Attribution License (https://creativecommons.org/licenses/by/4.0), which permits unrestricted use, distribution, and reproduction in any medium, provided the original work is properly cited. 
size, and dual-gradient structure of both grain size and twin thickness that are spatially varied, shown in Figures 1A-1D. While the extensively studied chemical gradients, including graded distributions in the phase, solid-solution concentration, and chemical components in biological materials [1], will not be discussed in this paper. Besides the types of basic structural components, their spatial distribution is another key characteristic of GNS, which differs from homogeneous or random distribution of the structural components. Accordingly, the gradient structure composed of the above-mentioned structural components can be either concentrated only on the sample surface (Figure 1E) or filled in the whole sample-level with monotonous (Figure 1F), symmetric (Figure 1G), or multi-periodic spatial variations (Figure 1H). Here, structural gradient, $s$, a quantitative factor to describe the spatial distribution degree of the GNS, is defined as the increment in hardness per unit thickness along the gradient direction in Figure 1F. The multiple structural components and distribution characteristics offer a spectrum of feasible pathways toward tailor-designing mechanical properties of GNS materials.

More importantly, we would like to emphasize that only when the characteristic size of any structural component refines as small as possible (that is so-called "go to nano") and the multi-scaled hierarchies (or properties) discrepancy of sample-level gradient structure becomes as large as possible (i.e., the length scales span six to seven orders of magnitude), the advantages of gradient nanostructures as well as the novel deformation mechanisms can be more significantly achieved or be activated.

The typical processing techniques used to synthesize GNS metals contain top-down methods via plastic deformation and bottom-up approaches via chemical (such as electrodeposition) or physical techniques. Surface mechanical treatment procedures, including surface mechanical attrition treatment, surface mechanical grinding treatment, and shot peening [12], have been well-developed to introduce surface gradient nanograined (GNG) structure (Figures 1A and 1E). However, the GNG layers introduced by these surface manufacturing approaches are universally located on the sample surfaces with a limited total volume fraction of $20 \%-25 \%$, wherein high-density dislocations prevail.

Electrodeposition technique is known for controllably synthesizing GNS samples with gradient volume fraction of $100 \%$, and low initial dislocation density [13]. For instance, pure Ni samples possessing a GNG structure with a monotonic change in the grain size up to three orders of magnitude were prepared by electrodeposition, where the degree of grain size gradient can be accurately tuned [14]. Gradient nanotwinned (GNT) $\mathrm{Cu}$ samples [10] were also fabricated by direct-current electrodeposition with a controllable patterning of homogeneous NT components from monotonous, symmetric periodic to multiperiodic spatial variations, as shown in Figures $1 \mathrm{~F}-1 \mathrm{H}$. These GNT samples exhibit a dual gradient distribution in both grain size and twin thickness (Figure 1D). Although exhibiting precise controllability, this chemical approach can only be used to introduce GNS structures in a few pure metals with low deposition rates. Other universal and efficient innovative synthesis methods are in urgent need for preparing varied GNS; more importantly, they should be widely feasible for more engineering alloys.

Recently, a simple, yet efficient cyclic-torsion (CT) treatment without any surface tooling was successfully developed to introduce novel sample-level gradient nanoscale stable dislocation structures with low-angle boundary misorientations (GDS, Figures $1 \mathrm{C}$ and $1 \mathrm{G}$ ) with a length scale spanning six magnitudes from millimeter to nanoscale in engineering materials, such as 304 stainless steel [15] and high entropy alloy (HEA) [11]. In particular, the initial grain structure, including grain size and morphology, is unchanged from the surface to the core, which is fundamentally distinct from the GNG structure with severely-refined grain 


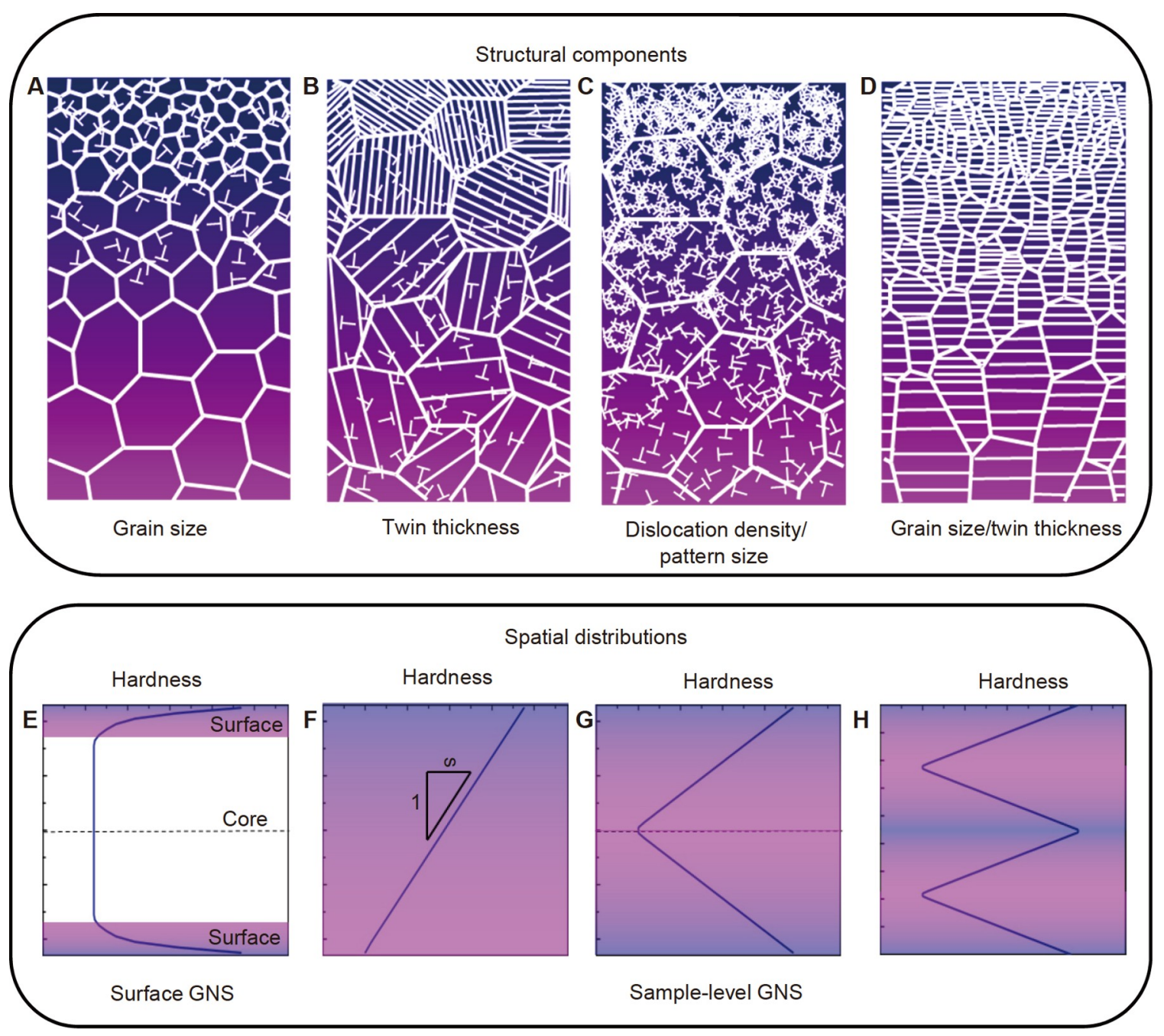

Figure 1 Structural components and spatial distributions in the GNS materials. Structural components contain grain size (A), deformation twin thickness (B), dislocation density or pattern size (C), and both the grain size and twin thickness (D). Spatial distributions include the GNS either in the sample surface (E) or in the sample-level with monotonous (F), symmetric (G), or multi-periodic spatial variation (H).

size. Owing to its controllably-tuned variable torsion parameters, such as torsion angle amplitude and torsion number, this new promising paradigm for tailoring properties by engineering stable gradient-dislocation patterns at the nanoscale can be potentially applicable to any metallic materials dominated by dislocation mechanisms.

Both structural component and spatial distributions play vital roles in determining the mechanical properties of the gradient nanostructure. The tensile stress-strain curves of interstitial-free (IF) steel with a surface GNG layer is shown in Figure 2A, as an example [9]. Due to the presence of surface GNG layer (with limited volume fraction of $25 \%$ ), the yield strength of GNG/CG IF steel doubles, whereas its ductility is slightly reduced, compared with that of the coarse-grained (CG) counterparts (Figures 2A and 2B). Similar phenomenon was universally observed in other GNG metals and alloys, such as $\mathrm{Cu}, \mathrm{Ni}$, and 316 steel [12]. However, owing to limited GNG volume fraction and structural gradient, the strength of these samples with a GNG/CG architecture roughly follows the rule-of-mixture according to that of the GNG surface layers and 

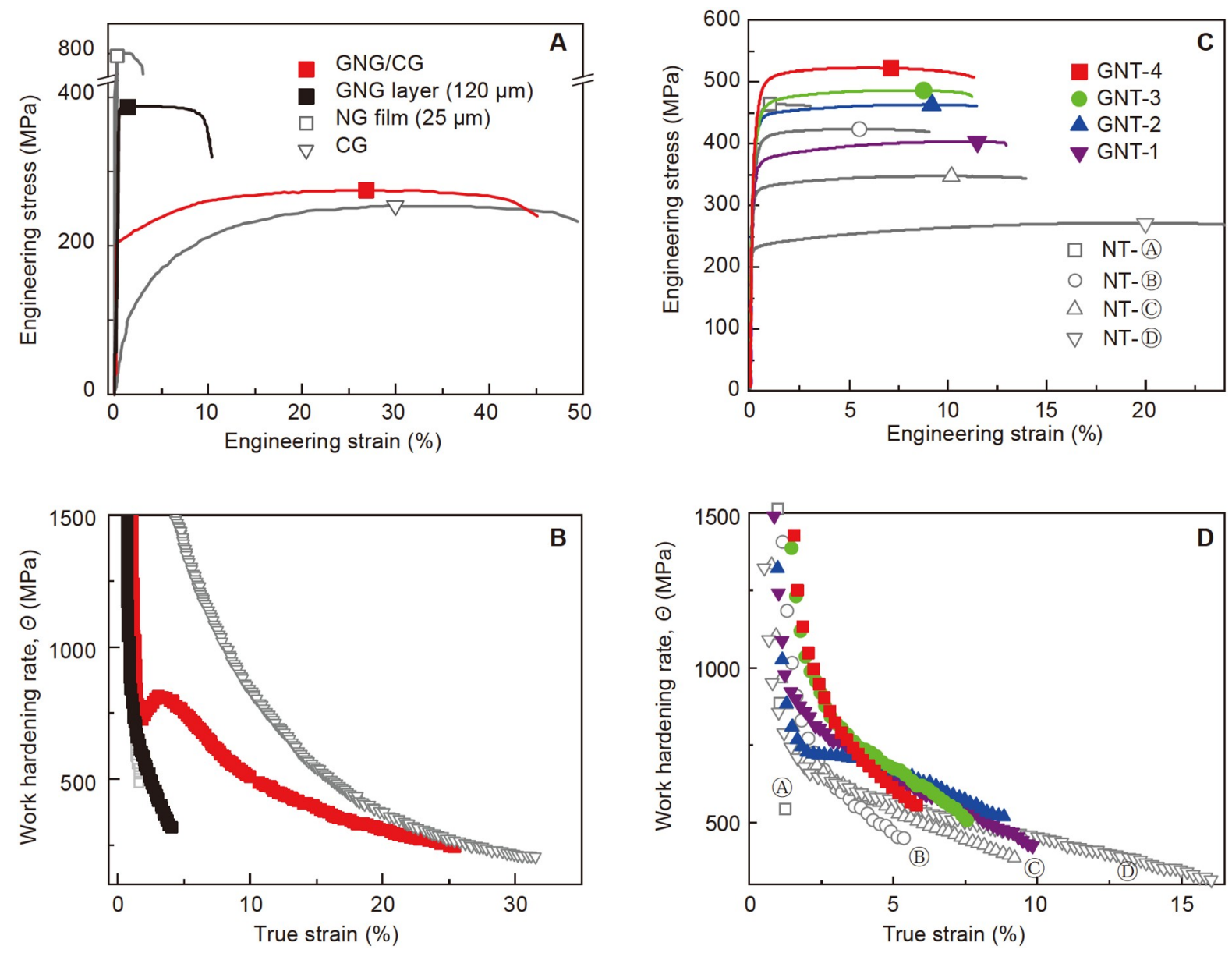

Figure 2 Superior mechanical properties of GNS materials. Tensile engineering stress-strain relations (A, C) and variation in work hardening rate with respect to true strain (B, D) of GNG IF steel (A, B) and GNT Cu (C, D) samples, compared with their homogeneous components. (A, B) adapted with permission from Ref. [9], PNAS; (C, D) adapted with permission from Ref. [10], AAAS.

CG substrate core. Meanwhile, the work hardening and tensile plasticity of GNG/CG samples strongly rely on their CG substrate (Figure 2B). A mechanically driven grain boundary migration process with grain coarsening or shear band and resultant softening were proposed to dominate the plastic deformation of the GNG structures [12].

The presence of nanoscale twin components remarkably elevates the mechanical properties of GNT $\mathrm{Cu}$ samples. Simultaneous enhancement in both strength and work hardening rate can be achieved by solely increasing the structural gradient, which even exceeds the strongest component, yet accompanied by a slight reduction in the uniform elongation, indicative of extra strengthening and work hardening (Figures $2 \mathrm{C}$ and 2D). Such an unprecedented strength-ductility combination primarily stems from the sample-level stable NT component with large structural gradients, which cannot be achieved by any other structure. Moreover, gradient order also affects the tensile properties, i.e., a higher strength can be achieved in GNT Cu with normal gradient order (hard surfaces and soft core), compared to those with reverse gradient order (soft surfaces and hard core) [16].

When sample-level gradient dislocation patterns (Figure 1C) are introduced in a single $f c c$ phase 
$\mathrm{Al}_{0.1} \mathrm{CoCrFeNi}$ HEA by CT treatment [11], it exhibits a three times higher yield strength meanwhile maintaining steady work-hardening, and comparable tensile plasticity, compared with its CG counterparts. Such an exceptional synergy of strength and ductility has not been possibly achieved in homogenous or heterogeneous structures and most existing metals and alloys with gradient nanograins or nanotwins.

During the deformation of GNS materials, the structural gradient induces plastic deformation incompatibility, i.e., a remarkably ordered, progressive deformation transformation from elasticity to plasticity due to the yield strength discrepancy among different structural components. The resultant deformation incompatibility is generally accommodated through the generation of geometry necessary dislocations (GNDs) based on the theoretical models [17-20], which further leads to a built-in gradient in both plastic strain and stress, as demonstrated by experiments [10], theories and modeling [4,21,22]. Such inhomogeneous deformation on the scale of the structural gradient fundamentally differs from the case in conventional metallic materials without gradient. In particular, some novel dislocation activities, interface-related behavior, and interactions between GNDs and interfaces have been observed.

For example, a brand new dislocation pattern with bundles of concentrated dislocations (BCDs) is formed and uniformly distributed in the grains of GNT $\mathrm{Cu}$ at a small tensile strain (Figure 3A) [10]. These BCDs consist of both model I and model II dislocations from different slip systems, illustrated by Thompson tetrahedron and marked by the blue and green arrows in Figure 3B. By contrast, only mode II (green) dislocations are detected in the homogeneous NT component under the same loading condition. Coincidentally, a novel Shockley partial associated with stacking-faults-induced plasticity (SFIP) dominated strengthening mechanism, rather than the traditional full dislocations, is reported in the deformed GDS HEA [11]. After an initial tensile strain as small as 3\%, highly dense micrometer-length SF bundles in Figure 3C, composed of sub-10 nanometer SFs and twins (Figure 3D), are progressively activated to mediate the plastic deformation of GDS HEA.

Without a doubt, either the presence of BCDs with ultrahigh density of dislocation in GNT samples or high density of tiny SFs in GDS samples are products of the deformation of the gradient nanostructure and also products of GNDs assisted deformation. These novel dislocation patterns in the GNS microstructure can act as strong obstacles to further dislocation slips and the sustainable sources for dislocation nucleation and storage. These distinctive structural component-dependent deformation mechanisms are crucial to maintain structural stability across multiple-scales and suppress softening. Macroscopically, the sample-level orderly and progressive plastic strain in GNS samples effectively relaxes the local stress concentration between adjacent components of different length sizes and suppresses the strain localization, enabling different structural components of gradient nanostructure sample to plastic deform coherently, even at a higher overall stress level. As a result, extra strengthening and extra work hardening or an unprecedented better combination of strength and ductility can be achieved in both GNT and GDS materials. This effect will become more pronounced when the structure gradient becomes larger.

Despite the considerable progress in the processing and mechanics of GNS metals and alloys, there are still several open issues and challenges ripe for future experimental, analytical, and modeling studies. Further innovation in processing techniques is desired for precisely tailoring gradient nanostructures with multiple structural components and spatial gradients. The development of more cost-effective, yet efficient techniques with scale-up capability for GNS engineering materials is necessary for promoting their industrial production and application. 

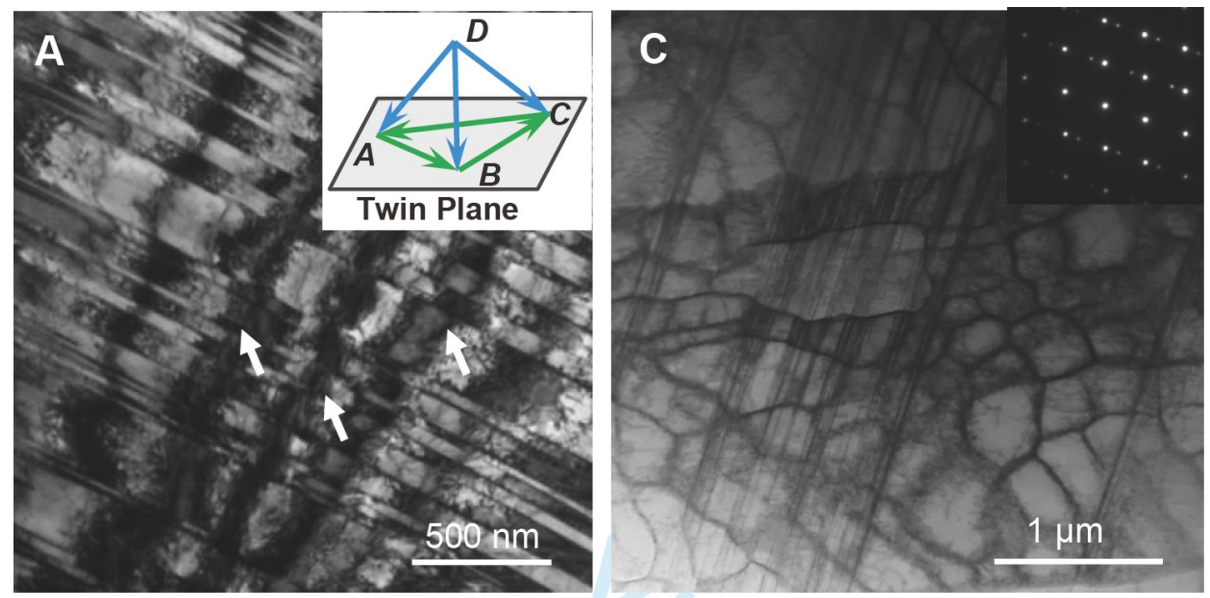

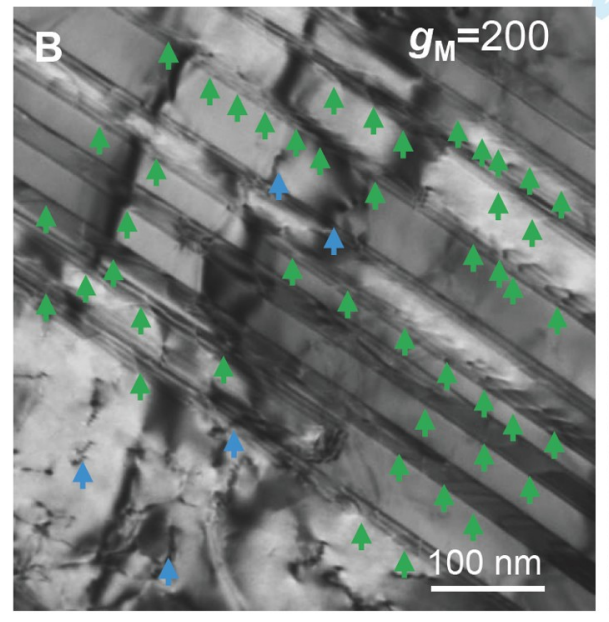

Bundle of concentrated dislocations

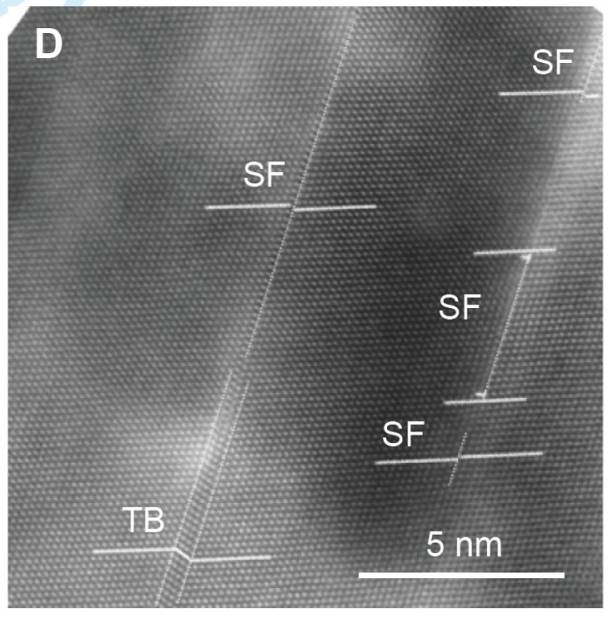

Stacking faults induced plasticity

Figure 3 Structural gradient induced novel deformation mechanisms. BCDs in GNT $\mathrm{Cu}$ at $1 \%$ strain, indicated by the white arrows (A), which are identified by two-beam diffraction using vectors of $g_{\mathrm{M}}=200$ (B). Highly dense micrometer-length SF bundles in the GDS HEA at $3 \%$ strain $(C)$, which are composed of numerous sub-10 nanometer SFs and tiny TBs (D). The inset in (C) is the corresponding selected area electron diffraction patterns containing parallel streaks from SFs. (A, B) adapted with permission from Ref. [10], AAAS; (C, D) adapted with permission from Ref. [11], AAAS.

Since the current design and optimization of gradient nanostructures remain largely empirical, it becomes urgent to develop quantitative theoretical and computational modeling frameworks for designing and optimizing multiscale structural parameters to achieve targeted mechanical properties. Multiscale modeling, atomistic simulation, and theoretical studies should be strengthened to reveal the novel deformation mechanism and address the role of structural gradient played on the novel mechanical responses. More multiscale advanced microstructural characterizations, including three-dimensional high-resolution transmission electron microscopy, in-situ synchrotron X-ray diffraction, and neutron diffraction characterizations, are expected and to be combined to explore the complicated structural and inhomogeneous stress/strain evolutions spanning different length scales.

Revealing the intrinsic mechanical properties, especially the service performance including fatigue, fracture, creep, corrosion, and wear, of various gradient nanostructures are an intriguing unexplored issue. Also, decoupling the multiple microstructural parameters, such as structural component, length scale, spatial 
distribution, structural gradient, and the volume fraction, on the mechanical behaviors of GNS metals and alloys is essential for further performance optimization. However, these related in-depth researches remain scarce. Finally, quantifying correlations among hierarchical gradient nanostructures, deformation mechanism, and damage behavior are essential for advancing the potential engineering applications of GNS materials.

\section{Data availability}

All data generated or analyzed during this study are included in this published article.

\section{Funding}

This work was supported by the National Natural Science Foundation of China (51931010, 92163202, 52122104, and 52071321), the Key Research Program of Frontier Science and International Partnership Program (GJHZ2029), and the Youth Innovation Promotion Association, Chinese Academy of Sciences (2019196).

\section{Author contributions}

L.L. initiated and supervised the project. Q.P. and L.L. contributed to the discussions and wrote the manuscript.

\section{Conflict of interest}

The authors declare no conflict of interest.

\section{References}

1 Suresh S. Graded materials for resistance to contact deformation and damage. Science 2001; 292: $2447-2451$.

2 Fang TH, Li WL, Tao NR, et al. Revealing extraordinary intrinsic tensile plasticity in gradient nano-grained copper. Science 2011; 331: 1587-1590.

3 Wei Y, Li Y, Zhu L, et al. Evading the strength-ductility trade-off dilemma in steel through gradient hierarchical nanotwins. Nat Commun 2014; 5: 3580.

4 Li X, Lu L, Li J, et al. Mechanical properties and deformation mechanisms of gradient nanostructured metals and alloys. Nat Rev Mater 2020; 5: 706-723.

5 Roland T, Retraint D, Lu K, et al. Fatigue life improvement through surface nanostructuring of stainless steel by means of surface mechanical attrition treatment. Scripta Mater 2006; 54: 1949-1954.

6 Pan Q, Lu L. Improved fatigue resistance of gradient nanograined metallic materials: Suppress strain localization and damage accumulation. Scripta Mater 2020; 187: 301-306.

7 Lu L, Pan Q, Hattar K, et al. Fatigue and fracture of nanostructured metals and alloys. MRS Bull 2021; 46: 258-264.

8 Kou H, Lu J, Li Y. High-strength and high-ductility nanostructured and amorphous metallic materials. Adv Mater 2014; 26: 5518-5524.

9 Wu X L, Jiang P, Chen L, et al. Extraordinary strain hardening by gradient structure. Proc Natl Acad Sci USA 2014; 111: 7197-7201.

10 Cheng Z, Zhou H, Lu Q, et al. Extra strengthening and work hardening in gradient nanotwinned metals. Science 2018; 362: eaau1925.

11 Pan Q, Zhang L, Feng R, et al. Gradient cell-structured high-entropy alloy with exceptional strength and ductility. Science 2021; 374: 984-989.

12 Lu K. Stabilizing nanostructures in metals using grain and twin boundary architectures. Nat Rev Mater 2016; 1: 16019.

13 Lu L, Shen Y, Chen X, et al. Ultrahigh strength and high electrical conductivity in copper. Science 2004; 304: 422-426. 
14 Lin Y, Pan J, Zhou H F, et al. Mechanical properties and optimal grain size distribution profile of gradient grained nickel. Acta Mater 2018; 153: 279-289.

15 Pan Q, Guo S, Cui F, et al. Superior strength and ductility of 304 austenitic stainless steel with gradient dislocations. Nanomaterials 2021; 11: 2613.

16 Cheng Z, Lu L. The effect of gradient order on mechanical behaviors of gradient nanotwinned Cu. Scripta Mater 2019; 164: $130-134$.

17 Ashby MF. The deformation of plastically non-homogeneous materials. Philos Mag-J Theor Exp Appl Phys 1970; 21: 399-424.

18 Fleck NA, Muller GM, Ashby MF, et al. Strain gradient plasticity: Theory and experiment. Acta Metall Mater 1994; 42: 475-487.

19 Nix WD, Gao H. Indentation size effects in crystalline materials: A law for strain gradient plasticity. J Mech Phys Solids 1998; 46: 411-425.

20 Gao H, Huang Y, Nix WD, et al. Mechanism-based strain gradient plasticity? I. Theory. J Mech Phys Solids 1999; 47: 1239-1263.

21 Zeng Z, Li X, Xu D, et al. Gradient plasticity in gradient nano-grained metals. Extreme Mech Lett 2016; 8: $213-219$.

22 Zhang Y, Cheng Z, Lu L, et al. Strain gradient plasticity in gradient structured metals. J Mech Phys Solids 2020; 140: 103946. 